\title{
Estudio de la demanda insatisfecha para el rediseño curricular de la carrera de contabilidad y auditoría, universidad nacional de Chimborazo en el Ecuador
}

\section{Study of the unsatisfied demand for the curricular redesign of the accounting and audit career, national university of Chimborazo in Ecuador}

Arellano Cepeda Otto

Coronel Sánchez Jhonny Mauricio

Cáceres Vargas Lisbeth Matilde

Zavala Heredia Jhony Rodrigo

Universidad Nacional de Chimborazo, Ecuador

Autor para correspondencia: areotto@gmail.com

Fecha de recepción: 08 de Mayo de 2017 - Fecha de aceptación: 10 de Agosto de 2017

Resumen: El Rediseño curricular como estrategia de gestión, propicia la acreditación de las Universidades en el Ecuador a fin de mejorar la calidad educativa y desarrollar nuevas competencias en los futuros profesionales. El estudio tiene el objetivo de rediseñar el currículo de la carrera de Contabilidad y Auditoría Contador Público Autorizado, acorde con el marco constitucional, legal y reglamentario vigente, en función a la determinación de la pertinencia y las demandas y necesidades de la sociedad. Como metodología se usa el método deductivo y la investigación es de tipo descriptivo. Se realiza una evaluación curricular a fin de establecer los perfiles profesionales que sirven para determinar las competencias. Se aplica el cuestionario para determinar la demanda insatisfecha a una muestra de 448 empresas de una población de 10.333 empresas en la zona 3. Los resultados muestran que existe una demanda insatisfecha, lo que permite describir el Rediseño de la Carrera de Contabilidad y Auditoría de la Universidad Nacional de Chimborazo Ecuador, de acuerdo al contexto jurídico (Plan Nacional del Buen Vivir, contexto regional (Zona 3) y los objetivos de la Educación Superior Nacional (Direccionamiento de la SENESCYT).

Palabras Clave: perfil profesional; empresas; calidad educativa; demanda; oferta

\begin{abstract}
The curricular redesign as a management strategy, promotes the accreditation of universities in Ecuador in order to improve the quality of education and develop new skills in future professionals. The purpose of the study is to redesign the curriculum of Accounting and Auditing Accountant, in accordance with the constitutional, legal and regulatory framework in force, depending on the determination of the relevance and the demands and needs of society. As a methodology, the deductive method is used and the research is descriptive. A curricular evaluation is carried out in order to establish the professional profiles that serve to determine the competences. The questionnaire is applied to determine the unsatisfied demand for a sample of 448 companies from a population of 10,333 companies in zone 3 . The results show that there is an unsatisfied demand, which allows to describe the Redesign of the Accounting and Auditing Career of the National University of Chimborazo Ecuador, according to the legal context (National Plan for Good Living, regional context (Zone 3) and the objectives of National Higher Education (Addressing SENESCYT).
\end{abstract}

Key words: professional profile; companies; educational quality; demand; offer 


\section{Introducción}

\section{Antecedentes de la investigación}

En 1969, se crea en la ciudad de Riobamba la Facultad de Ciencias de la Educación Filosofía y Letras como una Extensión de la Universidad Central del Ecuador con las Escuelas de: Educación Técnica, Comercio y Administración, Física y Matemática, Química y Biología, Ciencias Sociales, Educación Física, Literatura y Castellano, Idiomas, Filosofía; las mismas que otorgaban títulos de tercer nivel como Licenciado en Ciencias de la Educación en las diferentes especialidades.

La Universidad Nacional de Chimborazo es una persona jurídica, de derecho público, sin fines de lucro, autónoma, creada mediante Ley No. 98, publicada en el Suplemento del Registro Oficial No. 771 del 31 de agosto de 1995; su domicilio es la ciudad de Riobamba; sus siglas son UNACH; se rige por la Constitución de la República del Ecuador, Ley Orgánica de Educación Superior, su Reglamento, leyes conexas, Estatuto, Reglamentos y Resoluciones que expidan el Consejo de Educación Superior y la Universidad. Es una Institución de calidad y reconocimiento social en el Sistema de Educación Superior, que oferta sus carreras de pregrado en la modalidad presencial y semipresencial. El Honorable Consejo Universitario, el 23 de julio de 1997 crea el Programa Académico de Auditoria y Contabilidad. El 13 de mayo de 1998, el Consejo Universitario cambió la denominación de Programa Académico a Escuela de Auditoria y Contabilidad, formando parte de la Facultad de Ciencias Políticas y Administrativas. Desde su creación hasta el año 2003, la Carrera otorgó el título de Licenciado en Auditoría y Contabilidad, Contador Público Autorizado (C.P.A.), con un periodo de estudio de 4 años; a partir de del año lectivo 2003-2004 se ofertó el título de Ingeniero en Contabilidad y Auditoría, C.P.A., en 5 años de estudio hasta la actualidad. De conformidad con lo que dispone la Ley Orgánica de Educación Superior, la modalidad de estudios se modificó del sistema anual al sistema semestral a partir de septiembre del 2011, misma que se encuentra actualmente en un proceso de finalización.

Por tanto, el estudio tiene el objetivo de: "Rediseñar el currículo de la carrera de Contabilidad y Auditoría Contador Público Autorizado, acorde con el marco constitucional, legal y reglamentario vigente, en función a la determinación de la pertinencia y las demandas y necesidades de la sociedad”, en relación a lo Académico, Investigación, Vinculación, y Titulación (Art. 21,22,23 Reglamento de Régimen Académico), con un enfoque basado en competencias a partir de un planteamiento de un perfil profesional técnico- científico, que permite la planificación, evaluación y el desarrollo de competencias que respondan a las necesidad de los empleadores. Se considera que H1. La pertinencia del Rediseño está relacionada por la cantidad de empresas existentes y H2. La demandad insatisfecha determina el Rediseño Curricular.

\section{Marco Teórico}

El marco teórico responde a una revisión jurídica, teórica y filosófica que fundamenta el Rediseño curricular.

\section{a) Marco Legal o Jurídico del Rediseño curricular}


El rediseño curricular responde a los principios establecidos en la art. 351 de la Constitución Política de la República del Ecuador, la Ley Orgánica de Educación Superior, el Reglamento de Régimen Académico del Consejo de Educación Superior (CES), el Reglamento de Presentación y Aprobación de Carreras y Programas de las Instituciones de Educación Superior, Reglamento de Armonización de la Nomenclatura de Títulos Profesionales y Grados Académicos que Confieren las Instituciones de Educación Superior del Ecuador.

La Ley Orgánica de Educación Superior, da paso al marco normativo, el cual propone, organizar, regular y controlar las obligaciones y derechos a la educación Superior, dentro de parámetros comunes para todo el país, pero al mismo tiempo respeta la diversidad existente y fomenta la innovación educativa y la participación de los actores en su proceso; en consecuencia se consideran los principales artículos de este ley. Art. 1, 2, (Ley orgánica de Educación Superior, 2010). Además, el Estatuto Universitario (2013) establece en su Art. Nro. 1 que "La Universidad Nacional de Chimborazo es una institución de educación superior, con personería jurídica, sin fines de lucro, autónoma, de derecho público, creada mediante Ley No. 98, publicada en el Suplemento del Registro Oficial No. 771, del 31 de agosto de 1995, su domicilio principal es la ciudad de Riobamba; sus siglas son UNACH. Se rige por la Constitución de la República del Ecuador, la Ley Orgánica de Educación Superior, su Reglamento, otras leyes conexas, el presente Estatuto, los Reglamentos y Resoluciones que expidan el Consejo de Educación Superior; el Consejo de Evaluación, Acreditación y Aseguramiento de la Calidad de la Educación Superior; y, la Secretaría Nacional de Educación Superior, Ciencia, Tecnología e Innovación y la Universidad".

\section{b) Aspectos Teóricos del Rediseño}

La teoría que fundamenta el Rediseño tiene como base en documentos internos del CES publicados por Elizabeth Larrea de Granados (2015) como: 1) El currículo de la Educación Superior desde la Complejidad sistémica; 2) Modelo de organización del conocimiento por dominios científicos, tecnológicos y humanísticos, 3) Nuevas tensiones y funciones de la educación a tomar en cuenta en la construcción de los perfiles de los docentes, 4) Unidad Curricular de Titulación. Además, el rediseño viene fundamentado por las teorías del aprendizaje recogidas por Schunk (2012) siendo ellas las siguientes:

La teoría conductista, según Zapata (2016, pag.7 y 8) se da sus inicios a principios del siglo XX. Esta teoría, enfoca la psicología desde el mundo científico y educativo considerada como una ciencia natural más, está basada fundamentalmente en los cambios observables en la conducta del individuo, se enfoca en la repetición de patrones de conducta observables y medibles de un individuo, de manera que estos se ejecutan automáticamente, se toma animales como un punto de partida para la investigación, trata de explicar el comportamiento como una serie de estímulos y respuestas. Los representantes más importantes son: Pavlov (1927), Watson (1828), Skinner (1957), Hull (1940); así, Pavlov (1927) considera conceptos, como: el estímulo incondicionado, respuesta Incondicionada, estimulo neutro, estimulo condicionado, y respuesta condicionada; en cambio, para Schunk (2012, pág. 72), Watson crea el modelo de condicionamiento de Pavlov donde propone transformar la psicología en ciencia, alejándose de los enfoques estructurales y funcionalistas, conceptos como alma, mente conciencia, asumió el esquema E-R estímulo - respuesta. Skinner (1957) “centra su atención en las respuestas 
voluntarias, teoría del condicionamiento, que realiza el sujeto de experimentación, el conductismo debe identificar qué factores ambientales influye en la conducta, la recompensa y el castigo pasan a ocupar un lugar esencial en los esquemas conductistas, sus estudios se basan en el Condicionamiento Instrumental u operante, describe modelos, como: refuerzo positivo, refuerzo negativo, entrenamiento por omisión, y castigo" (Schunk, 2012, pág. 72).

La Teoría Cognitivista, surge en los años 60 y es una reacción a los limitantes del conductismo, el modelo de aprendizaje ya no es animal si no ordenador, la inteligencia artificial, la mente seria desde este punto de vista un instrumento que procesa la información, se acentúa los procesos más complejos en la solución de problemas, la formación de conceptos del procesamiento de información, sus representantes más importantes son: Piaget (1947), Bruner (1941), Ausubel (1950) y Vigotski (1925). Se impulsa el crecimiento de una Didáctica y Diseño Curricular centrado en lo Cognitivo.

La Teoría Constructivista, tiene "sus raíces históricas y filosóficas en los escritos de Bredo (1997) y a Packer y Goicochea (2000)" (Schunk, 2012, pág. 230); en esta corriente la persona adquiere y genera conocimiento en función de sus experiencias anteriores, una construcción que es resultado de la interacción de factores que intervienen día a día, el conocimiento no es una copia de la realidad si no una construcción del ser humano, que se realiza con los esquemas que la persona ya posee (conocimientos previos), que los construyo en su relación con el medio que lo rodea, y el contexto de su vida. Todo aprendizaje constructivo supone una construcción que lo realiza a través de un proceso mental que conlleva a la adquisición de un conocimiento nuevo el mismo que es acumulativo, y la posibilidad de construirlo y adquirirlo como una nueva competencia que le permite generalizar y aplicarlo a lo ya conocido en una nueva situación.

El Aprendizaje basado en Competencias, según García Retana (2011, pág. 6) hace referencia a varios autores como: (Akhyar, 2010), (Ortega, 2008), (Aguerrondo, 2009), (Alonso y Gallegos, 2010), quienes mencionan conceptos del modelo educativo basado en Competencias, conceptualizando que el desarrollo de las competencias es el esfuerzo por integrar el proceso educativo de manera que se pueda garantizar que se ha alcanzado la formación completa, y los alumnos han desarrollado estilos propios de aprendizaje, se plantea el reto de estimular la creatividad, innovación y potencialidad del ser humano. En ella es importante que el diseño curricular considere las competencias, siendo que la curricula "es un proceso educativo integral que mantiene interdependencia con otros contextos y/o ámbitos" (Yanes (2005) en García (2011, pág. 7), donde "la universidad tiene la misión de formar en primer lugar como persona, y en segundo lugar como profesional, que desempeñará un trabajo para el que está siendo preparado...." (Villa Sánchez \& Villa Leicea, 207, pág. 17) por consiguiente el estudiante aprende y actúa éticamente, en función social, alcanza un conocimiento (conceptual, procedimental y actitudinal) y este se convierte en una herramienta que le permite mostrar qué puede hacer con ese conocimiento. A esto es a lo que se llama una competencia. En este sentido la actitud es parte de la competencia desarrollada. En la formulación de los aprendizajes es muy importante tomar en cuenta, el entorno o el contexto, en el que se llevara a cabo la experiencia de aprendizaje. 
El Aprendizaje basado en retos, es una nueva tendencia educativa del Aprendizaje, la cual forma parte de una perspectiva más general del Aprendizaje Vivencial. Primero se abordan elementos del Aprendizaje Vivencial, para después enfocar la discusión hacia el aprendizaje centrado en la solución de retos (Observatorio de innovación Educativa del Tecnológico de Monterrey, 2015, pág. 4). Según López (2015) el Aprendizaje Basado en Retos tiene sus raíces en el Aprendizaje Vivencial, el cual tiene como principio fundamental que los estudiantes aprenden mejor cuando participan de forma activa en experiencias abiertas de aprendizaje, que cuando participan de manera pasiva en actividades estructuradas. Asimismo, según Association for Experiential Education (2015) el "Aprendizaje Vivencial ofrece oportunidades a los estudiantes de aplicar experiencias de aprendizaje diseñadas, con actividades de reflexión, análisis crítico y síntesis, las experiencias de lo que aprenden en situaciones reales donde se enfrentan a problemas, descubren por ellos mismos, prueban soluciones e interactúan con otros estudiantes dentro de un determinado contexto" (Moore, 2013, pág. 4), como también menciona Akella (2010) que el Aprendizaje Vivencial es un enfoque holístico integrador del aprendizaje, que combina la experiencia, la cognición y el comportamiento. En el campo de la educación, destacados psicólogos y filósofos como John Dewey (1884), Jean Piaget (1947), William Kilpatrick (1928), Carl Rogers (1942) y David Kolb (1984) realizaron importantes aportes a las teorías del aprendizaje a través de la experiencia, plasmado en el modelo Kolb (1984). En García (2015) el Aprendizaje Basado en Retos es una técnica de enseñanza-aprendizaje colaborativa en la que se plantea una situación problemática relacionada con el entorno físico o social. A menudo utiliza escenarios de casos ficticios; su objetivo no es resolver el problema en sí, sino usarlo para el desarrollo del aprendizaje, el producto final puede ser tangible o bien, una propuesta de solución al problema.

\section{Materiales y Métodos}

Se considera el método inductivo y el tipo de investigación descriptivo. Como unidad de estudio se constituyen las empresas de la Zona 3, provincia de Chimborazo de la República del Ecuador. Se aplica como instrumento de recogida de información el cuestionario y como técnica la encuesta a una muestra de 448 empresas (muestreo pro balístico) en una población total de 10.333 empresas aplicada durante los meses de marzo a mayo del 2015 (ver tabla 1) a fin de determinar la demanda insatisfecha a partir de la demanda laboral y la oferta académica de la regional zona 3 .

Tabla 1. Distribución del Universo

\begin{tabular}{lllll}
\hline Antón & Personas Naturales & Sociedades & $\begin{array}{l}\text { Total } \\
\text { Empresas }\end{array}$ & \% Participación \\
\hline Alausi & 93 & 419 & 512 & $5 \%$ \\
Chambo & 47 & 88 & 135 & $1 \%$ \\
Chunchi & 48 & 166 & 214 & $2 \%$ \\
Colta & 35 & 498 & 533 & $5 \%$ \\
Cumanda & 90 & 123 & 213 & $2 \%$ \\
Guamote & 70 & 468 & 538 & $5 \%$ \\
Guano & 132 & 280 & 412 & $4 \%$ \\
Pallatanga & 48 & 132 & 180 & $2 \%$ \\
\hline
\end{tabular}




\begin{tabular}{lllll}
\hline Penipe & 20 & 118 & 138 & $1 \%$ \\
Riobamba & 3007 & 4451 & 7458 & $72 \%$ \\
Total General & 3.590 & 6.743 & 10.333 & $100 \%$ \\
\hline
\end{tabular}

Fuente: Elaboración propia con base en datos del SRI - 2015

El tamaño de muestra es de 448 empresas en función a la fórmula 1. Para poblaciones finitas de acuerdo a los datos que aparecen en la tabla 2.

Fórmula 1. Determinación del tamaño de muestra para poblaciones finitas

$$
n=\frac{N \sigma^{2} Z^{2}}{(N-1) e^{2}+\sigma^{2} Z^{2}}
$$

Tabla 2. Datos

\begin{tabular}{cl}
\hline $\mathrm{N}$ & 10333 \\
\hline$\sigma 2$ & 0,5 \\
$\mathrm{Z}$ & 2,58 \\
$\mathrm{E}$ & $5,96 \%$ \\
$\mathrm{~N}$ & 448
\end{tabular}

Fuente: Elaboración propia con base en datos del SRI, 2015

\section{Resultados}

\section{Determinación de la pertinencia para el Rediseño Curricular}

Los resultados de la investigación muestran que el rediseño de la Carrera de Contabilidad y Auditoría está relacionada por la cantidad de empresas, puesto que el $20 \%$ de las empresas en la Provincia del Chimborazo son públicas y el $80 \%$ son privadas (ver tabla 3), lo que significa que la región se caracteriza por un mercado ofertante de empresas con carácter privado, por tanto existe pertinencia.

Tabla 3. Tipo de empresa/institución

\begin{tabular}{lll}
\hline Variable & Frecuencia & Porcentaje \\
\hline Pública & 91 & $20 \%$ \\
Privada & 357 & $80 \%$ \\
TOTAL & 448 & $100 \%$ \\
\hline
\end{tabular}

Fuente: Encuesta Empresarios y empleadores, 2015

\section{Determinación de la demanda insatisfecha para el Rediseño Curricular}

Demostrar H2. Que la demandad insatisfecha determina el Rediseño Curricular de la Carrera de Contabilidad y Auditora en la Universidad Nacional de Chimborazo, implico la determinación de la demanda empresarial, la oferta empresarial y la demanda insatisfecha, como se presenta a continuación:

\section{Determinación de la demanda empresarial de profesionales del Área Contable}


La región de la Provincia de Chimborazo, por los resultados de la investigación presenta una alta demanda de profesionales contadores por parte de los empleadores (empresa) ya que en el $70 \%$ de la empresas encuestadas mencionan que son muy necesarios para el desarrollo del país; lo que significa que existe demanda laboral para profesionales del área (ver gráfico 1) de la carrera de contabilidad y Auditoría.

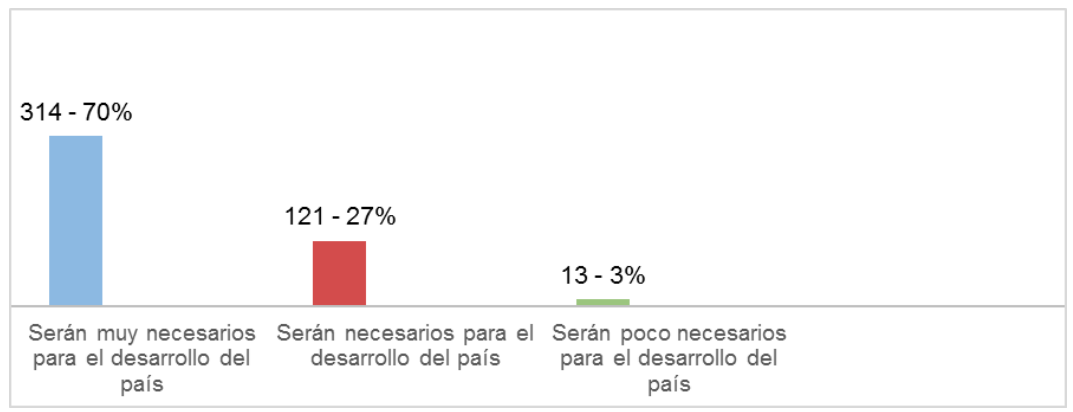

Gráfico 1. Demanda empresarial de contadores

Fuente: Elaboración propia con base en Encuesta Dirigido a Empresarios, 2015

Asimismo, las principales áreas de ocupación demandas por las empresas corresponden a puestos de trabajos relacionados con la actividad de la Contabilidad en un 96\%, Tributación $64 \%$, Auditoría 48\%, presupuestos un 38\%, Tesorería un 34\%, Pagaduría un 32\% y Cumplimiento un $27 \%$, Bodega un $17 \%$ y Otros un $10 \%$, que cubren las necesidades ocupacionales de las empresas (Ver tabla 4 y grafico 3.), lo que significa que existe mayor demanda en puestos de trabajo relacionados con la: Contabilidad, Tributación, Auditoría. Lo que demuestra que existe una alta demanda en el mercado laboral de profesionales del área contable.

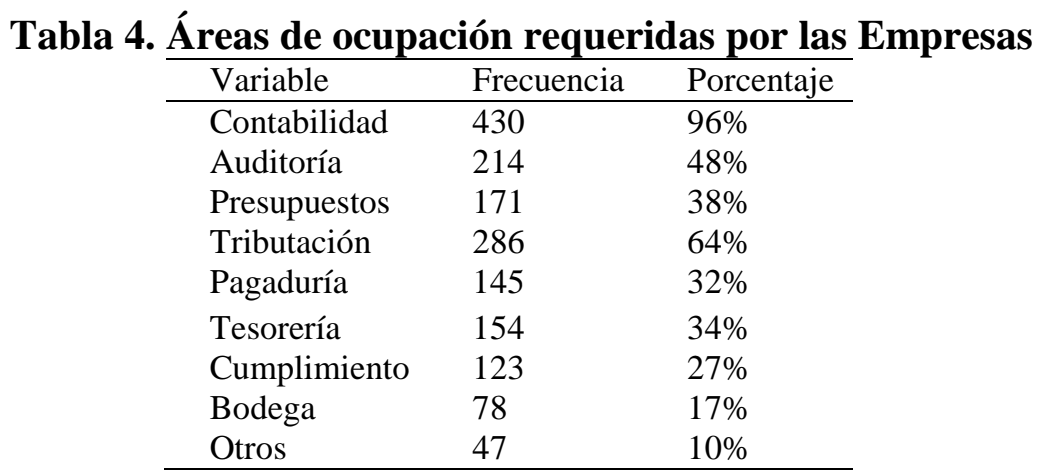

Fuente: Encuesta Empresarios y empleadores, 2015 


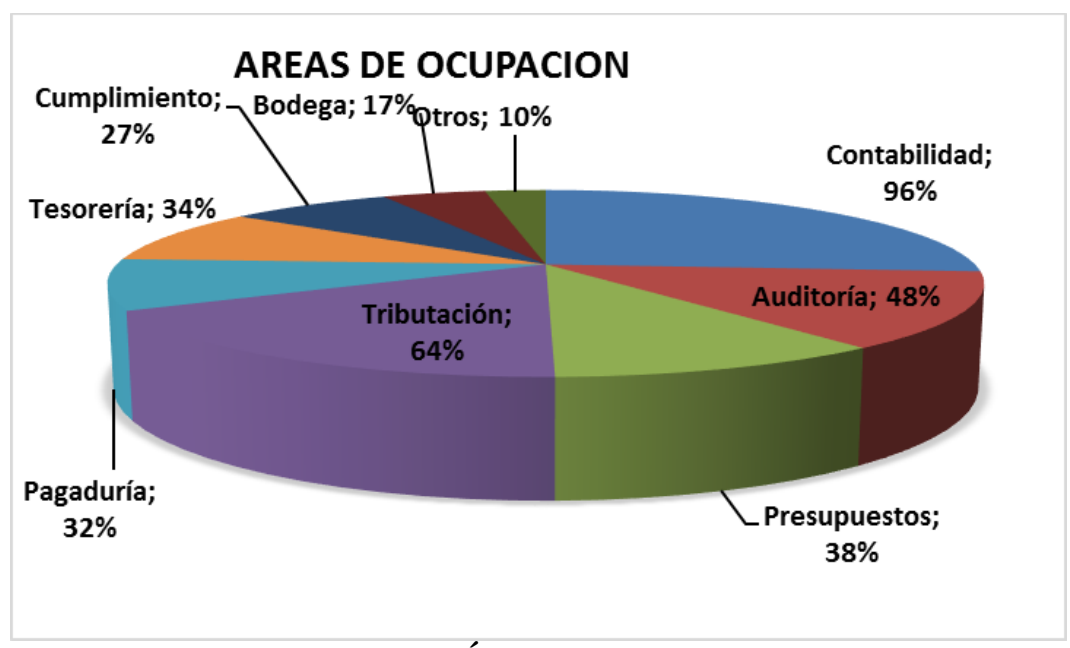

Gráfico 3. Áreas de ocupación Fuente: Tabla 4

\section{Determinación de la oferta Académica de profesionales de la Carrera de Contabilidad y Auditoria}

Los resultados de la investigación de acuerdo al reporte de demanda y oferta académica del Sistema de Nivelación y Admisión, de la Secretaria de Educación Superior, Ciencia, Tecnología e Innovación, al 24 de agosto del 2015, muestran que en el sub área del conocimiento, Educación Comercial y Administración, Carrera de Contabilidad y Auditoría desde el año 2014 al 2015 (Ver gráfico 2) se mantienen constantes por la asignación a la mencionada área (ver tabla 5.)

Tabla 5. Oferta Académica por Sub área del Conocimiento marzo 2014 marzo 2015

\begin{tabular}{llll}
\hline OFERTA ACADEMICA & mar-14 & sep-14 & mar-15 \\
\hline Periodismo e información & 35 & 30 & 35 \\
Industria y producción & 35 & 32 & 35 \\
Artes & 40 & 30 & 35 \\
Protección del medio ambiente & 35 & 35 & 35 \\
Ingeniería y profesiones afines & 40 & 32 & 35 \\
Informática & 40 & 32 & 35 \\
Agricultura, silvicultura y pesca & 40 & 32 & 35 \\
Derecho & 80 & 70 & 70 \\
Arquitectura y construcción & 75 & 134 & 65 \\
Educación comercial y administración & 145 & 105 & 105 \\
Ciencias sociales y del comportamiento & 170 & 160 & 140 \\
Medicina & 245 & 250 & 175 \\
Formación de personal docente y ciencias & 400 & 280 & 245 \\
\hline
\end{tabular}

Fuente: SNNA, 2015

\section{Determinación de la demanda insatisfecha y proyección de la demanda profesional}


La demanda insatisfecha profesional viene dada por la diferencia entre la demanda laboral por parte de las empresas y la oferta por parte la carrera de Contabilidad y Auditoría. Los resultados de la investigación determinan que existe una demanda insatisfecha de 67 profesionales para el año 2017, incrementándose en promedio en un $177 \%$ hasta el año 2021 tomando como año base el año 2017 (Ver tabla 6).

Tabla 6. Demanda insatisfecha de profesionales

\begin{tabular}{clllc}
\hline Año & $\begin{array}{c}\text { Número De } \\
\text { Estudiantes Titulados }\end{array}$ & $\begin{array}{c}\text { Número De Nuevas } \\
\text { Empresas } \\
\text { A }\end{array}$ & $\begin{array}{c}\text { Demanda Insatisfecha De } \\
\text { Profesionales } \\
\text { A-B=C }\end{array}$ & $\begin{array}{c}\text { Tasa De } \\
\text { Crecimiento }\end{array}$ \\
\hline 2016 & 73 & 76 & -3 & $100 \%$ \\
2017 & 35 & 102 & -67 & $134 \%$ \\
2018 & 38 & 128 & -90 & $173 \%$ \\
2019 & 38 & 154 & -116 & $216 \%$ \\
2020 & 35 & 180 & -145 & $263 \%$ \\
2021 & 30 & 206 & -176 & \\
\hline
\end{tabular}

Elaborado: Comisión de Rediseño Carrera de Contabilidad con base en Encuesta

El incremento de la demanda de profesionales en el área contable y de auditoria se debe a un incremento en la creación de nuevas empresas que alcanzaría de 493 en el año 2017 a 905 para el año 2021 (ver tabla 7) en la Provincia de Chimborazo, Cotopaxi, Pastaza y Tungurahua como se puede observar en el gráfico 4.

Tabla 7. Proyección de creación de nuevas empresas

\begin{tabular}{llllllllll}
\hline Provincia & 2013 & 2014 & 2015 & 2016 & 2017 & 2018 & 2019 & 2020 & 2021 \\
\hline Cotopaxi & 29 & 32 & 52 & 61 & 73 & 85 & 97 & 109 & 121 \\
Chimborazo & 7 & 29 & 110 & 152 & 204 & 256 & 308 & 360 & 412 \\
Pastaza & 15 & 17 & 24 & 28 & 33 & 38 & 43 & 48 & 53 \\
Tungurahua & 54 & 66 & 122 & 149 & 183 & 217 & 251 & 285 & 319 \\
& 105 & 144 & 308 & 390 & 493 & 596 & 699 & 802 & 905
\end{tabular}

Fuente: Elaboración propia con base en datos de la Superintendencia de Compañías y Seguros, 2013 -2015

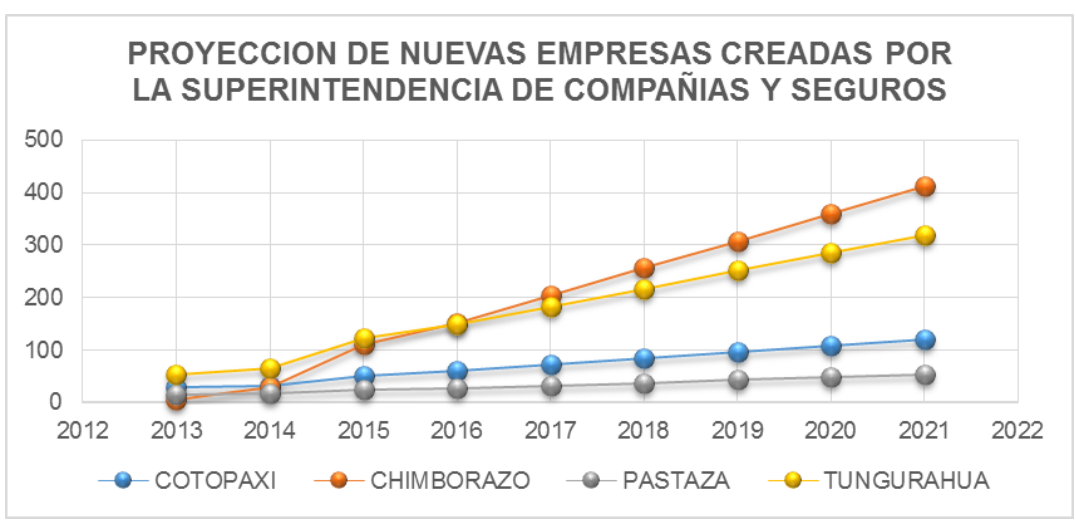

Gráfico 4. Proyección de creación de nuevas empresas

Fuente: Tabla 7

Lo que significa que la demanda insatisfecha determina la realización del rediseño ya que existe un incremento en la demanda hasta el año 2022. 


\section{El Rediseño Curricular de la Carrera de Contabilidad y Auditoría}

\section{a) Importancia de la Carrera de Contabilidad y Auditoría en el Rediseño}

El desarrollo económico empresarial en Ecuador se ha localizado en las ciudades de Guayaquil, Quito, Cuenca y Manta, Ambato lo que permite un comercio equilibrado a nivel interno, como el desarrollo de actividades comerciales a nivel externo por la disponibilidad de infraestructura, portuaria, aérea, además de recibir por parte del gobierno a la generación comercial a fin de fortalecer las regiones y zonas que no tuvieron un desarrollo económico sustentable. El Plan Nacional de Desarrollo 2013 -2017 denominado como el Plan Nacional del Buen Vivir, definido en el artículo N 280 de la Constitución de la República del Ecuador es un "instrumento al que se sujetarán las políticas, programas y proyectos público, la programación y ejecución el presupuesto del Estado y la inversión y la asignación de los recursos públicos; y coordinar las competencias exclusivas entre el Estado central y los gobiernos autónomos descentralizados. Su observancia será de carácter obligatorio para el sector público e indicativo para los demás sectores", lo que permite a nivel de las Instituciones de Educación Superior formar profesionales en el campo de la Contabilidad y Auditoría, que respondan a las necesidades de empleabilidad en sus áreas específicas.

Desde este contexto, la carrera de Contabilidad y Auditoría responde a las necesidades locales de la región del Chimborazo principalmente (ver Tabla 7), lo que significa que la carrera aporta de forma positiva en el desarrollo local dotando de profesionales que ocupan puestos relacionados en el área, como la de: Contadores, Auditores, presupuestos, tributación, y otros.. Sin embargo, no existe un registro específico de la empleabilidad y actividades que realizan los profesionales, la mayor parte de los profesionales Contables prestan sus servicios en Asesoría tributaria y contable en oficinas propias, en los Gobiernos Autónomos Descentralizados Parroquiales, Municipales y Provinciales, en organizaciones de Economía Popular y Solidaría y en empresas de sector público y privado, por lo que no se puede restringir la importancia de las funciones que realizan los profesionales en este campo.

\section{b) Núcleos Básicos de la Carrera para el Rediseño Curricular}

La carrera de Contabilidad a fin de responder a las necesidades de la empresas contempla núcleos de formación profesional como (ve grafico 7) la de: 1) Contabilidad, con el propósito de que el profesional realice funciones de registro y síntesis de los efectos financieros de las operaciones que realiza una entidad económica, así como de los hechos económicos externos que le afectan para informar a los interesados y tomen decisiones oportunas y acertadas; 2) Auditoría, con el objeto de que el profesional debe estudiar el conjunto de normas, procesos y procedimientos de auditoría, que se aplican para evaluar y analizar las operaciones administrativas y financieras de una organización, a fin de obtener evidencia suficiente y opinar sobre el estado que guardan estas funciones con relación a los principios, normas y procedimientos establecidos en la profesión contable, emitiendo un informe que contenga, comentarios, conclusiones y recomendaciones o informando a los interesados mediante un dictamen que les permita tomar decisiones al respecto; 3)Tributación, con la finalidad donde el profesional maneje la naturaleza de las obligaciones fiscales de las personas naturales y 
jurídicas, para financiar el gasto público, según lo establece la legislación ecuatoriana; 4) Finanzas, con el objetivo de realizar actividades relacionadas con el intercambio y manejo de capital. Como una parte de la economía ya que tienen que ver con las diferentes maneras de administrar dinero en situaciones particulares y específicas. Las finanzas permiten estudiar múltiples aspectos y elementos relacionados con todo el proceso de la obtención y administración del dinero o capital. Buscan mejorar las fuentes de las que se obtiene dinero y busca optimizar su utilización, que se puede derivar en su gasto o inversión; 5) Investigación, en ella se reconoce la importancia del desarrollo de la investigación a nivel, local, zonal, nacional e internacional, por tanto se considera un eje transversal en la formación del licenciado en Contabilidad y Auditoría, en la ejecución de los procesos sustantivos de la carrera. En este proceso de investigación, los docentes en su función de educadores, tutores y asesores, se constituyen en un ente importante para el desarrollo del pensamiento científico de los estudiantes, cuyo producto final establece la transformación de la realidad en concordancia con los cambios acelerados que suceden en el país y el mundo globalizado.

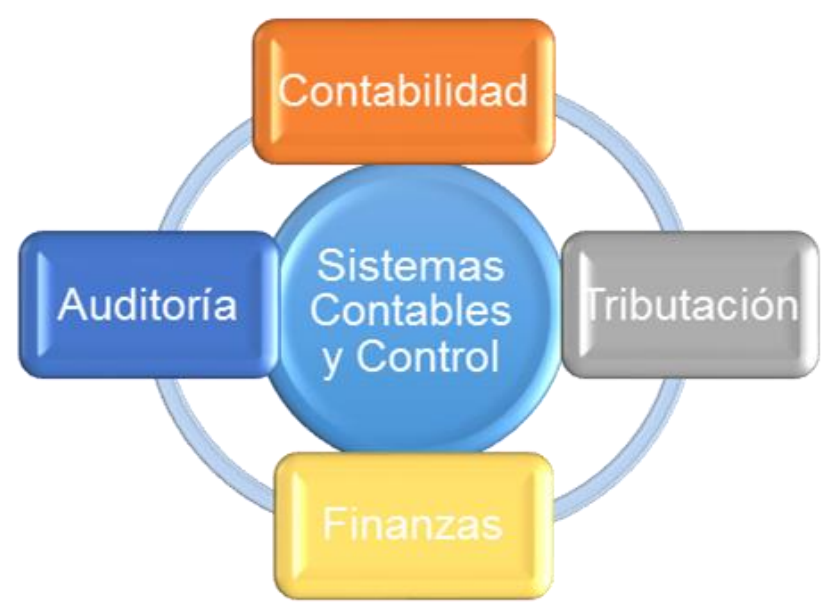

Gráfico 6. Núcleos básicos de la Carrera de Contabilidad y Auditoria

Fuente: Elaboración propia con base en al rediseño de la Carrera de Contabilidad y Auditoría, 2015

\section{c) Distribución de las asignaturas en el Rediseño Curricular}

La distribución de las asignaturas corresponde a Unidades de Organización Curricular establecidas en el Reglamento de Régimen Académico CES, 2015. De un total de 51 asignaturas, 17 asignaturas corresponden en la Unidad Básica, 24 asignaturas en la Unidad Profesional y 10 asignaturas en la unidad de Titulación (Ver tabla 8).

Tabla 8. Distribución de asignaturas cursos o equivalentes

\begin{tabular}{lllll}
\hline $\begin{array}{c}\text { Unidad De } \\
\text { Organización } \\
\begin{array}{l}\text { Curricular } \\
\text { Académicos }\end{array}\end{array}$ & $\begin{array}{c}\text { Asignaturas, Cursos O } \\
\text { Equivalentes Por Periodo } \\
\text { Académico }\end{array}$ & $\begin{array}{l}\text { Asignaturas Por } \\
\text { Unidad De } \\
\text { Organización } \\
\text { Curricular }\end{array}$ & $\begin{array}{c}\text { \% Por Unidad De } \\
\text { Organización } \\
\text { Curricular }\end{array}$ \\
\hline Unidad Básica & Primero & 6 & 17 & $33,33 \%$ \\
& Segundo & 5 & & \\
& Tercero & 6 & & \\
\hline
\end{tabular}




\begin{tabular}{lllcc}
\hline Unidad & Cuarto & 6 & 24 & $47,06 \%$ \\
Profesional & Quinto & 6 & & \\
& Sexto & 6 & & \\
& Séptimo & 6 & 10 & $19,61 \%$ \\
Unidad De & Octavo & 6 & & $100 \%$ \\
Titulación & Noveno & 4 & 51 & \\
Total & & 51 & & \\
\hline
\end{tabular}

Fuente. Malla Curricular de la Carrera, 2016

\section{d) Perfil de ingreso considerado en el Rediseño Curricular}

El perfil de ingreso a la Carrera de contabilidad y Auditoría viene precedida al cumplimiento de niveles mínimos de aptitudes académicas, conocimientos, habilidades y destrezas, que demuestren las competencias básicas adquiridas en el bachillerato, en relación a las áreas: Linguística, Científico - Técnico, Socio - Humanística y Razonamiento Lógico, para evaluar la comprensión y aplicación de las nociones aprendidas; tales como:

- Capacidad de lectura comprensiva.

- Capacidad de razonamiento lógico.

- Capacidad de expresión oral y escrita.

- Capacidad de análisis y síntesis.

- Capacidad de autoestima y predisposición para culminar la Carrera.

- Habilidades en el manejo de las Tecnologías de la Información y Comunicación.

- Práctica de valores éticos y morales.

\section{e) Perfil de egreso}

El perfil del egreso responde a:

- Ejercer su profesión con responsabilidad ética y vocación de servicio dentro de un marco de principios y valores humanísticos de respeto hacia la sociedad y el medio ambiente.

- Utiliza el emprendimiento e innovación con enfoque en el desarrollo de la matriz productiva que le permita generar empleo en la pequeña y mediana empresa y contribuir al cumplimiento del Plan Nacional del Buen Vivir.

- Elabora informes contables y financieros aplicando disposiciones legales y normas internacionales de información financiera, relacionados al funcionamiento de las empresas e instituciones públicas y privadas.

- Utiliza las Tics como herramienta de gestión para la elaboración de los estados financieros y los dictámenes de auditoría, así como en sus actividades particulares y empresariales.

- Identifica Calcula los elementos del costo de producción y prestación de servicios, aplicando procedimientos, sistemas, políticas contables y gerenciales para la toma de decisiones 
- Aplica indicadores financieros y de gestión para diseñar planes efectivos de soluciones de casos en el ámbito de la contabilidad, auditoría y finanzas que permita mejorar la posición socio económica de las organizaciones.

- Descubre y previene fraudes y delitos financieros en el desempeño de su práctica profesional.

\section{f) Perfil profesional}

El profesional en Contabilidad y Auditoría tendrá la capacidad de desarrollar actividades:

- El Contador - Auditor aplica principios morales y éticos tales como la integridad, objetividad, imparcialidad, independencia, honestidad, honradez, confidencialidad.

- Fomenta e innova emprendimientos, analiza, sintetiza, trabaja en equipo, negocia y toma decisiones, organiza, maneja los recursos financieros y económicos de las empresas con responsabilidad, contribuyendo a cumplir con los Objetivos Plan Nacional del Buen Vivir.

- Diseña e implementa sistemas de información donde se aplique la teoría contable y el registro de operaciones acorde con los principios, normas de contabilidad generalmente aceptada, Leyes y Reglamentos afines a su profesión mediante el uso de herramientas computarizadas.

- Organiza, coordina y supervisa el funcionamiento económico y administrativo en empresas públicas y/o privadas mediante el manejo integral de la contabilidad con el uso de las Tics.

- Elabora, analiza e interpretar Estados Financieros para apoyar a una correcta y eficiente toma de decisiones.

- Planifica y ejecuta Auditorias Financieras, Administrativas, de Gestión e Informáticas acorde con las disposiciones legales y las Normas de Auditoria Generalmente Aceptadas

- Audita las áreas Funcionales de la empresa siguiendo la filosofía de mejora continua, diseña, implementa y evalúa Sistemas de Control Interno para un adecuado uso de recursos.

\section{Discusión}

La formación del profesional Contable responde a las necesidades y requerimientos de la provincia y la Zona 3, convertida en el centro de acopio agrícola y enclave comercial a escala nacional, el estudio de la pertinencia está vinculada a la vocación productiva, agropecuaria, manufacturera, ambiental, energética y de servicios de la zona, ésta encaminada a ofrecer una carrera profesional a una población mestiza, indígena, afroecuatorianos, blancos y de los pueblos y nacionalidades indígenas; el rediseño y pertinencia es una oferta con una sólida formación académica, investigativa, vinculación articulada con prácticas profesionales, en empresas públicas y privadas. La Carrera tiene como objeto de estudio los Sistemas contables y Control, la formación profesional de Contadores - Auditores, con dominios en las disciplinas básicas del conocimiento Contabilidad, Tributación, Auditoría y Finanzas, en concordancia con las nuevas tendencias nacionales e internacionales para la creación y construcción del conocimiento en contextos de investigación e innovación, con énfasis en el manejo eficiente de los recursos económicos y financieros. Elabora los estados financieros de acuerdo a la norma nacional e 
internacional vigente, ejecuta auditorias, utiliza métodos y técnicas cuantitativas en el procesamiento y análisis de información financiera.

La Carrera de Contabilidad y Auditoría, incorpora nuevas competencias a la Sociedad del Conocimiento como desafío para superar las barreras intelectuales creadas en la formación ocupacional, acordes a los señalamientos del Plan Nacional del Buen Vivir 2013-2017, la Agenda Para la Transformación Productiva 2010 - 2013, la propuesta de cambios en el conocimiento de la Matriz Productiva, Agenda Zonal y Provincial 2011; Plan de Desarrollo Chimborazo Minga por la Vida 2015 - 2020, las corrientes y requerimientos de los actores y sectores Internacionales. En este contexto la importancia del Contador - Auditor radica en un alto nivel de profesionalismo a fin de vincularse al progreso de la comunidad, contribuyendo de esta manera al crecimiento económico de la sociedad ya que "el gran salto de una sociedad primaria-extractivita a una sociedad del conocimiento sólo es posible si actuamos en distintos ámbitos, principalmente en la generación y transferencia de conocimiento, y en la formación de personas e instituciones de excelencia. En este contexto, estamos creando el ambiente ideal para incrementar la productividad sistémica del país y desarrollar nuevas áreas de investigación como son: Bio-Ciencias, Tecnologías de la Información y Comunicación, Nano-Ciencias, Energías Alternativas, entre otras..." (Espinoza, 2013).

\section{Conclusiones}

El estudio de pertinencia y la determinación de la demanda insatisfecha de la carrera de Contabilidad y Auditoría, obtuvo herramientas para la construcción de un nuevo currículo que se ajusta a los requerimientos y necesidades de la sociedad, promoviendo la integración de los saberes ancestrales y la intercultaralidad, con formación humana y ética, capaz de responder al mundo laboral.

La nueva Malla Curricular se fundamenta en los requerimientos de los sectores y actores, y en la solución de los nodos críticos que resuelve la carrera, dando énfasis en la praxis profesional, la fundamentación teórica, la investigación, y complementando con itinerarios multiprofesionales, dando formación a los futuros profesionales en Contabilidad y Auditoría de acuerdo a necesidades laborales de la zona 3 y de todo el país.

El Rediseño curricular de la carrera responde a las necesidades empresariales y a las nuevas competencias requeridas por el estado, la administración tributaria, las empresas públicas y privadas, para esto se determinaron el objeto de estudio, los objetivos de formación de la carrera, el perfil profesional, elementos que están alineados a las nuevas tendencias nacionales e internacionales.

La construcción de la Misión, Visión, objetivos, perfil de egreso, perfil profesional de Contador, competencias generales, se articulan a los objetivos del Plan Nacional del Buen Vivir, la problemática socioeconómica, y requerimientos laborales de la zona 3.

\section{Bibliografía}

Agenda para la transformación productiva territorial Chimborazo. (mayo 2011). 
Agenda para la transfromación productiva 2010- 2013. (s.f.). Consejo Sectorial de la Producción.

Asamblea Constituyente. (2008). Constitución de la República del Ecuador. Montecristi.

Belletine, O., \& Ordoñez, A. (2013). Ecuador del País de los recursos al país del conocimiento. Grupo Faro.

Bravo Valdiviezo, M. (2011). Contabilidad General (Décima edición ed.). Loja: Nuevo Día.

Consejo de Educación Superior. (2013). Reglamento de Régimen Académico. Quito: Consejo de Educación Superior.

Consejo de Educación Superior. (2014). Reglamento de presentación y aprobación de carreras y programas de instituciones de educación superior (RPC - SO- 32 Nro. 358-2014 ed.). Quito, Ecuador: Consejo de Educación Superior. Recuperado el 2015

Consejo de Educación Superior. (2015). Reglamento de armonización de la nomenclatura de títulos profesionales y grados académicos que confieren las instituciones de educación superior del Ecuador. (CES- RPC-SO-27-Nro. 289-2014 ed.). Quito: Consejo de Educación Superior.

Espinoza, A. X. (2013). Hacia el país del conocimiento 2013.

Estatuto de la Universidad Nacional de Chimborazo. (2013). Riobamba, Ecuador.

Franco Ruiz, R. (Febrero de 2002). Consideraciones acerca de la Investigación Contable y sus Desarrllos Teóricos. Reflexiones Contables(Segunda edición), 102.

García Retana, J. Á. (septiembre - diciembre de 2011). Modelo Educativo Basado en Competencias: Importacnia y Necesidad. Revista Electrónica "Actualidades Investigativas en Educación", 11(3), 26.

Larrea de Granados, E. (2015). El currículo de la educación superior desde la complejidad.

Larrea de Granados, E. (2015). Modelo de organización del conocimiento por dominios científicos, Tecnológicos y humanísticos.

Larrea de Granados, E. (2015). Nuevas tensiones y funciones de la educación a tomar encuenta en la construcción de los perfiles de los docentes.

Ley orgánica de Educación Superior. (2010). Quito, Ecuador: Registro Oficial. Recuperado el 2015

López García, J. C. (2015). http://www.scoop.it/t/jualop. Obtenido de http://www.scoop.it/t/jualop.

Ministerio Coordinador de Conocimiento y Talento Humano. (s.f.). Nota Técnica Nro. 2 Balanza del Conocimiento. Ministerio Coordinador de Conocimiento y Talento Humano. 
Observatorio de innovación Educativa del Tecnológico de Monterrey. (01 de noviembre de 2015). http://www.eduteka.org/articulos/edutrends-10-2015. (eduteka) Recuperado el diciembre de 2015, de http://www.eduteka.org/articulos/edutrends-10-2015: www.eduteka.org

Pimienta Prieto, J. H. (2012). Estrategias de enseñanza-aprendizaje Docencia universitaria basada en competencias (Primera Edición ed.). México, México: Pearson Educación.

Plan de desarrollo Chimborazo Minga por la Vida 2005 - 2020. (2005). Riobamba.

Plan Nacional del Buen Vivir 2013 - 2017. (2013). Secretaria Nacional de Planificación y Desarrollo.

Reglamento de Régimen Académico de la Universidad Nacional de Chimborazo. (2015). Riobamba.

Schunk, D. H. (2012). Teorías del aprendizaje una perspectiva educativa (Sexta Edición ed.). México: Pearson Educación.

Villa Sánchez, A., \& Villa Leicea, O. (207). El aprendizaje basado en competencias y el desarrollo de la dimensión social en las universidades. Educar, 40, 15-48.

Zapata Ros, M. (2016). Teórias y modelos sobre el aprendizaje en entornos conectados y ubicuos bases para un nuevo modelo. Departamento de Computación, Universidad de Alcalá, España, 49.

Zapata Sánchez, P. (2011). Contabilidad General (Séptima edición ed.). (M. Chueco, Ed.) Bogotá, Colombia: McGrawHill Educación. 